where the last reduction was made by interchanging integrations in (23) and by comparing the result with (22). The interchange was permissible* because of our present hypotheses. Since $\left[z_{i}\left(x^{\prime}\right)-z_{i}(x)\right]$ is the $i$ th Fourier coefficient of the left side of (20), we have completed the proof of the theorem.

In a later paper the author will consider applications of the present results in the theory of functionals whose arguments are summable functions.

UNIVERSity OF Minnesota, December 15, 1920.

\title{
NOTE ON MINIMAL VARIETIES IN HYPERSPACE.
}

\author{
BY PROFESSOR C. L. E. MOORE.
}

(Read before the American Mathematical Society December 29, 1920.)

1. IT is known that a necessary and sufficient condition that a surface of two dimensions in hyperspace be minimal is the vanishing of the vector mean curvature. $\dagger$ It is the purpose of this note to show that mean curvature of a variety $V_{m}$ in a space of $n$ dimensions can be defined in the same way and that its vanishing is a necessary and sufficient condition that $V_{m}$ be minimal. I shall use the absolute calculus, since one of its chief merits is the ease with which invariants can be written down. In fact the very form of an expression shows whether or not it is invariant. Enough vector analysis is used to simplify the form of the expressions.

The variety $V_{m}$ can be written vectorially. in the form

Then

$$
y=y\left(x_{1}, x_{2}, \cdots, x_{m}\right) .
$$

$$
d s^{2}=d y \cdot d y=\sum_{1}^{m} a_{r s} d x_{r} d x_{s}
$$

If we write

$$
y_{r}=\frac{\partial y}{\partial x_{r}},
$$

* Cf. de la Vallée Poussin, loc. cit., p. 53.

†Wilson and Moore, "Differential geometry of two-dimensional surfaces in hyperspace," Proceedings of the Amer. Acad., vol. 52 (1916). 
we see at once that

(1)

$$
a_{r s}=y_{r} \cdot y_{s}
$$

Hence we may write

$$
d y \cdot d y=\Sigma y_{r} \cdot y_{s} d x_{r} d x_{s} .
$$

Differentiating (1) covariantly with respect to the first fundamental form,

$$
\varphi=\Sigma a_{r s} d x_{r} d x_{s},
$$

remembering that the first covariant derivative of the coefficients of the fundamental form is zero, we obtain

$$
y_{r t} \cdot y_{s}+y_{r} \cdot y_{s t}=0 \text {, }
$$

and since this relation holds for any $r, s$, we may write

$$
\begin{aligned}
& y_{t s} \cdot y_{r}+y_{t} \cdot y_{s r}=0 \\
& y_{r s} \cdot y_{t}+y_{r} \cdot y_{s t}=0
\end{aligned}
$$

The vector $y$ is a function of $x_{1}, x_{2}, \cdots, x_{m}$. Since the second covariant derivative of a function is symmetric, we have

$$
y_{r s}=y_{s r} \text {. }
$$

From the last four equations we have

$$
y_{r} \cdot y_{s t}=0, \quad r, s, t=1,2, \cdots m .
$$

These equations show that $y_{s t}$ is perpendicular to $y_{r}$, and it is therefore perpendicular also to the tangent $m$-space of $V_{m}$.

Since the $y_{r s}$ are vectors lying in the normal space, we can express them as linear functions of mutually perpendicular vectors in that space, which is of $n-m$ dimensions. Thus we find

$$
y_{r s}=b_{1 / r s} z_{1}+b_{2 / r s} z_{2}+\cdots+b_{(n-m) / r s} z_{n-m} .
$$

We have also the following relations:

$$
z_{i} \cdot z_{j}=0,(i \neq j) ; \quad z_{i} \cdot z_{i}=1, \quad z_{i} \cdot y_{s}=0 .
$$

Differentiating these covariantly, we have

$$
z_{i / s} \cdot z_{j}+z_{i} \cdot z_{j / s}=0 ; \quad z_{i / r} \cdot y_{s}+z_{i} \cdot y_{r s}=0
$$

Then, multiplying (3) through by $z_{i}$, we have

$$
z_{i} \cdot y_{r s}=b_{i / r s}=-z_{i / r} \cdot y_{s} \text {. }
$$


2. The Second Fundamental Forms.-The coefficients $b_{i / r s}$ are generalizations of the coefficients of the second fundamental form of a surface in $n$-space.* They may be taken as the coefficients of $n-m$ fundamental forms which we can combine into a vector fundamental form

$$
\psi=\Sigma y_{r_{s}} d x_{r} d x_{s}
$$

We now consider the $m$ systems of curves

$$
\lambda_{i}{ }^{(r)}=\frac{d x_{r}}{d s_{i}}, \quad(i=1,2, \cdots m)
$$

where $d s_{i}$ is the element of arc along the curve $\lambda_{i}$. Multiplying by the proper factor, we can make the quantities $\lambda_{i}$ satisfy the relation

$$
\Sigma_{r} \lambda_{i}{ }^{(r)} \lambda_{i / r}=1 .
$$

We shall now assume that this relation is satisfied. Such systems are called unit systems. The $\lambda$ 's are called the coordinates of the congruence. If the congruences are orthogonal, the coordinates satisfy the relations

$$
\begin{aligned}
& \Sigma \lambda_{i / r} \lambda_{j}{ }^{(r)}=\epsilon_{i j}, \\
& \Sigma \lambda_{i / r} \lambda_{i}{ }^{(s)}=\epsilon_{r s},
\end{aligned}
$$

where $\epsilon_{k l}=0$, if $k \neq l, \epsilon_{k k}=1$. In terms of the coordinates, we can write the coefficients of the first fundamental form as follows: $\dagger$

$$
a_{r s}=\Sigma_{i} \lambda_{i / r} \lambda_{i / s} .
$$

The coefficients of the second fundamental form can be written as follows:

$$
y_{r s}=\Sigma_{i, j} \omega_{i j} \lambda_{i / r} \lambda_{j / s}
$$

where $\omega_{i j}$ is a vector. The invariant vectors

$$
\xi_{i}=\Sigma_{r} \lambda_{i}{ }^{(r)} y_{r}=\Sigma_{r} \lambda_{i / r} y^{(r)}
$$

are mutually orthogonal unit vectors tangent to the curves whose covariant coordinates are $\lambda_{i / r}$. Solving these equations for $y_{r}$ and $y^{(r)}$ we find

$$
y_{r}=\Sigma_{i} \xi_{i} \lambda_{i / r}, \quad y^{(r)}=\Sigma \xi_{i} \lambda_{i}{ }^{(r)} .
$$

* See Wilson and Moore, loc. cit., p. 308.

$\dagger$ Wilson and Moore, loc. cit., §13, p. 289, and §\$26, 27, pp. 310-311. 
We now introduce Ricci's coefficients of rotation*

$$
\gamma_{h k l}=\Sigma_{r, s} \lambda_{h / r s} \lambda_{k}{ }^{(r)} \lambda_{l}{ }^{(s)} .
$$

Solving these equations for $\lambda_{h / r s}$, we find

$$
\lambda_{h / r s}=\Sigma_{i, j} \gamma_{h i j} \lambda_{i / r} \lambda_{j / s} \text {. }
$$

Substitution of this value in the covariant derivative of (7) gives

$$
\xi_{i / r}=\Sigma_{j} \omega_{i j} \lambda_{j / r}+\Sigma_{j, l} \gamma_{i j} \lambda_{l / r} \xi_{j} .
$$

The condition that the congruence $\lambda_{i}$ is a congruence of geodesics $\dagger$ is

$$
\gamma_{j i i}=0 \text {. }
$$

3. The Invariants $\omega_{i i}$. The curvature of a curve of the congruence $\lambda_{i}$ which passes through a given point is obtained by differentiating $\xi_{i}$ with respect to the arc length $s_{i}$. Thus we find

$$
C_{i}=\frac{d \xi_{i}}{d s_{i}}=\Sigma_{r} \frac{\partial \xi_{i}}{\partial x_{r}} \cdot \frac{\partial x_{r}}{\partial s_{i}}=\Sigma \xi_{i / r} \lambda_{i}{ }^{(r)},
$$

which, by (9), reduces to

$$
C_{i} \equiv \omega_{i i}-\Sigma_{j} \gamma_{j i i} \xi_{j} .
$$

The formula shows that the invariant vector $\omega_{i i}$ is the normal component of the curvature of the curve of the congruence in question. The tangential component of the curvature is

$$
\gamma=\Sigma_{j} \gamma_{j i i} \xi_{j} \text {. }
$$

The invariant $\gamma$ is then the geodesic curvature, $\ddagger$ since the curvature of the projection on the tangential space is the projection of the curvature. $\S$ If the congruence $\lambda_{i}$ is geodesic, $\gamma_{j i i}=0$. Hence the curvature of a geodesic is directed along the normal and is equal to $\omega_{i i}$.

An important invariant connected with any two differential forms is

$$
-\Sigma a^{(r s)} y_{r s}=\Sigma a_{r s} y^{(r s)}=\Sigma_{i j k r s} \omega_{i j} \lambda_{i / r} \lambda_{i / s} \lambda_{k}{ }^{(r)} \lambda_{k}^{(s)}=\Sigma \omega_{k k} .
$$

* Ricci and Levi-Civita, "Méthodes de calcul différentiel absolu," etc., Math. Annalen, vol. 54, p. 148.

+ Ricci and Levi-Civita, loc. cit., p. 148.

¥ Ricci and Levi-Civita, loc. cit., p. 154.

$\S$ Wilson and Moore, loc. cit., p. 320. 
Hence the sum of the normal curvature vectors of $m$ mutually orthogonal curves on the spread is constant. We shall define the mean curvature* $h$ by the relation

$$
m h=\Sigma \omega_{i i} \text {. }
$$

4. Minimal Varieties.-Applying the preceding formulas, we can now easily derive the condition that the volume of a variety of $m$ dimensions bounded by a variety of $m-1$ dimensions, chosen arbitrarily, shall be a minimum. The vector element of volume is

$$
P d x_{1} d x_{2} \cdots d x_{m}=\frac{\partial y}{\partial x_{1}} \times \frac{\partial y}{\partial x_{2}} \times \cdots \times \frac{\partial y}{\partial x_{m}} d x_{1} d x_{2} \cdots d x_{m} .
$$

The condition for a minimum is

$$
\delta \int(P \cdot P)^{\frac{1}{2}} d x_{1} d x_{2} \cdots d x_{m}=\int \frac{\delta P \cdot P}{(P \cdot P)^{\frac{1}{2}}} d V=\int \delta P \cdot M d V=0,
$$

where $M$ is the unit tangent $n$-space

Now

$$
M=\frac{P}{(P \cdot P)^{\frac{1}{2}}} .
$$

$$
\begin{aligned}
\delta P=\frac{\partial \delta y}{\partial x_{1}} \times \frac{\partial y}{\partial x_{2}} \times \cdots \times \frac{\partial y}{\partial x_{m}} & +\frac{\partial y}{\partial x_{1}} \times \frac{\partial \delta y}{\partial x_{2}} \times \cdots \times \frac{\partial y}{\partial x_{m}} \\
& +\cdots+\frac{\partial y}{\partial x_{1}} \times \frac{\partial y}{\partial x_{2}} \times \cdots \times \frac{\partial \delta y}{\partial x_{m}} .
\end{aligned}
$$

The condition for a minimum then becomes the vanishing of $n$ vector integrals. One of these integrals, after an integration by parts and the omission of the part which vanishes at both limits, becomes

$$
\begin{aligned}
\int\left(\frac{\partial \delta y}{\partial x_{1}}\right. & \left.\times \frac{\partial y}{\partial x_{2}} \times \cdots \times \frac{\partial y}{\partial x_{m}}\right) \cdot M d V \\
& =-\int \delta y \times \frac{\partial}{\partial x_{1}}\left(\frac{\partial y}{\partial x_{2}} \times \frac{\partial y}{\partial x_{3}} \times \cdots \times \frac{\partial y}{\partial x_{m}} \cdot M\right) d V .
\end{aligned}
$$

* This is the direct generalization of mean curvature as used in the theory of surfaces. There is, however, a mean curvature definable in terms of the first fundamental form, and this is the one used by Einstein. The definition used in this note depends on the space in which $V_{m}$ lies. 
The complete condition then becomes

$$
\begin{aligned}
\int \delta y & \times\left[\frac{\partial}{\partial x_{1}}\left(\frac{\partial y}{\partial x_{2}} \times \cdots \times \frac{\partial y}{\partial x_{m}} \cdot M\right)-\frac{\partial}{\partial x_{2}}\left(\frac{\partial y}{\partial x_{1}} \times \frac{\partial y}{\partial x_{3}}\right.\right. \\
\times & \left.\left.\cdots \times \frac{\partial y}{\partial x_{m}} \cdot M\right)+\cdots \pm \frac{\partial}{\partial x_{m}}\left(\frac{\partial y}{\partial x_{1}} \times \cdots \times \frac{\partial y}{\partial x_{m-1}} \cdot M\right)\right]=0 .
\end{aligned}
$$

Since $\delta y$ is arbitrary,

$$
\begin{aligned}
& \frac{\partial}{\partial x_{1}}\left(\frac{\partial y}{\partial x_{2}} \times \frac{\partial y}{\partial x_{3}} \times \cdots \times \frac{\partial y}{\partial x_{m}} \cdot M\right)-\frac{\partial}{\partial x_{2}}\left(\frac{\partial y}{\partial x_{1}} \times \frac{\partial y}{\partial x_{3}} \times \cdots\right. \\
&\left.\quad \times \frac{\partial y}{\partial x_{m}} \cdot M\right)+\cdots \pm \frac{\partial}{\partial x_{m}}\left(\frac{\partial y}{\partial x_{1}} \times \cdots \times \frac{\partial y}{\partial x_{m-1}} \cdot M\right)=0
\end{aligned}
$$

which on multiplying out reduces to

$\frac{\partial y}{\partial x_{2}} \times \frac{\partial y}{\partial x_{3}} \times \cdots \times \frac{\partial y}{\partial x_{m}} \cdot \frac{\partial M}{\partial x_{1}}-\frac{\partial y}{\partial x_{1}} \times \frac{\partial y}{\partial x_{3}} \times \cdots \times \frac{\partial y}{\partial x_{m}} \cdot \frac{\partial M}{\partial x_{2}}$

Now substituting

$$
+\cdots=0 \text {. }
$$

$$
\begin{aligned}
\frac{\partial y}{\partial x_{r}} & =y_{r}=\Sigma_{k} \xi_{k} \lambda_{k / r}, \\
M & =\xi_{1} \times \xi_{2} \times \cdots \times \xi_{m}, \\
\frac{\partial M}{\partial x_{i}} & =\frac{\partial \xi_{1}}{\partial x_{i}} \times \xi_{2} \times \cdots \times \xi_{m}+\xi_{1} \times \frac{\partial \xi_{2}}{\partial x_{i}} \times \xi_{3} \times \cdots \times \xi_{m}+\cdots,
\end{aligned}
$$

and using (9), the condition for a minimum becomes

$$
\left(\Sigma_{i} \omega_{i i}\right)\left|\lambda_{i / r}\right|=0 .
$$

The determinant $\left|\lambda_{i / r}\right|$ is equal to $\sqrt{a}$, where $a$ is the determinant of the first fundamental form, for from the expression $a_{r s}=\Sigma_{i} \lambda_{i / r} \lambda_{i / s}$ we have

$$
a=\left|a_{r s}\right|=\left|\Sigma_{i} \lambda_{1 / r} \lambda_{i / s}\right|=\left|\lambda_{i / r}\right| \cdot\left|\lambda_{i / s}\right|=\left|\lambda_{i / r}\right|^{2} .
$$

Hence the condition for a minimum is $\Sigma \omega_{i i}=0$.

The vanishing of the mean curvature vector is a necessary and sufficient condition that the variety be minimal. This is the same condition as that for a 2 -surface in hyperspace.

Massachusetts Institute of Technology, November 24, 1920. 